\title{
Structural energetics of serine protease inhibition*
}

\author{
Kenneth P. Murphy†, Brian M. Baker‡, Stephen P. Edgcomb and \\ James R. Horn \\ Department of Biochemistry, University of lowa, lowa City, IA 52242, USA
}

\begin{abstract}
We have investigated the binding of the serine protease inhibitor, turkey ovomucoid third domain (OMTKY3), to the serine protease, porcine pancreatic elastase (PPE), using isothermal titration calorimetry and structural energetics calculations. The calculations predict that the binding at $25^{\circ} \mathrm{C}$ is characterized by a negligible $\Delta H^{\circ}$, a large and positive $\Delta S^{\circ}$, and a large and negative $\Delta C_{\mathrm{p}}$, resulting in a large and favorable $\Delta G^{\circ}$. The experimental results indicate a significant contribution to the binding energetics from a change in the $\mathrm{pKa}$ of an ionizable group, presumably His57 of PPE. The resulting proton linkage is manifest in the observed $\Delta H^{\circ}$ and $\Delta C_{\mathrm{p}}$ of binding. However, a global analysis of binding data as a function of $\mathrm{pH}$, buffer, and temperature yields the intrinsic binding energetics as well as the energetics of proton binding to the ionizable group in the free and bound PPE. The experimentally determined intrinsic energetics and the calculated values are in very good agreement, suggesting that the structural energetics calculations may be a useful tool for understanding protein-protein interactions in solution.
\end{abstract}

\section{INTRODUCTION}

Nearly all biological processes require the recognition of a ligand by a biological macromolecule, a process known as molecular recognition. In many cases, this molecular recognition takes place between two protein molecules in a protein-protein interaction. A major goal of biophysical chemistry is the prediction of the affinity between a protein and a ligand based on knowledge of the three-dimensional structure. The ability to predict affinity based on structure serves as a basis for understanding structure-function relationships and for structure-based design of pharmaceuticals. Additionally, the affinity between a protein and a ligand is mediated by environmental variables such as temperature and $\mathrm{pH}$, and the dependence of the affinity on these variables must be understood.

The primary goal is to predict and understand the affinity constant, $K$, or, alternatively, the Gibbs energy of binding, $\Delta G^{\circ}$, which is related to the affinity constant as $\Delta G^{\circ}=-R T \ln K$, where $R$ is the gasconstant and $T$ is the absolute temperature, and many approaches exist which try to predict affinity at the level of $\Delta G^{\circ}$ [1-5]. However the Gibbs energy is comprised of both enthalpic and entropic contributions through the relationship $\Delta G^{\circ}=\Delta H^{\circ}-T \Delta S^{\circ}$. These contributions reflect the role of various interactions such as the hydrophobic effect, hydrogen bonding, and conformational entropy. If a prediction of $\Delta G^{\circ}$ for a particular system is in good agreement with experimental data it is difficult to tell if this is because the separate terms have been estimated correctly or if compensating errors cancel each

\footnotetext{
*Lecture presented at the 15th International Conference on Chemical Thermodynamics, Porto, Portugal, 26 July-1 August 1998, pp. 1167-1306.

$\dagger$ Correspondence: E-mail: k-murphy@uiowa.edu

$\ddagger$ Current address: Department of Molecular and Cellular Biology, Harvard University, 7 Divinity Ave., Cambridge, MA 02138, USA.
} 
other. Further, if the prediction is not in good agreement with the experimental data, it is difficult to know which part of the calculation needs refinement.

In contrast to attempts at directly predicting $\Delta G^{\circ}$, the method described here, structural energetics, attempts to predict $\Delta H^{\circ}$ and $\Delta S^{\circ}$ at some reference temperature, as well as the heat capacity change, $\Delta C_{\mathrm{p}}$, which determines the temperature dependence of $\Delta H^{\circ}$ and $\Delta S^{\circ}$. The $\Delta G^{\circ}$ (and $K$ ) can be calculated as a function of temperature from these parameters. If all three of the predicted terms, $\Delta H^{\circ}, \Delta S^{\circ}$ and $\Delta C_{\mathrm{p}}$, agree with experimental values, there is less likelihood of fortuitous agreement due to compensating errors. If there is disagreement between the calculated and experimental $\Delta G^{\circ}$, it can be ascertained where the difficulty lies. For example, disagreement between the predicted and experimental $\Delta S^{\circ}$ might suggest that the treatment of conformational entropy needs refinement.

This paper reviews recent studies in this laboratory on the binding of a protease inhibitor, turkey ovomucoid third domain (OMTKY3), and a serine protease, porcine pancreatic elastase (PPE) [6]. We have performed structural energetics calculations using a model of the complex between these proteins and compared the predicted energetics to the experimental values determined using isothermal titration calorimetry.

Elastase is a member of the serine protease family of enzymes which catalyze the cleavage of peptide bonds in proteins and polypeptides. Central to the function of these enzymes is the catalytic triad in the active site composed of a serine (Ser195), a histidine (His57), and an aspartic acid (Asp102) residue. In the course of catalysis, the proton on the hydroxyl of Ser195 is transferred to the side chain of His57 and the resulting positive charge is stabilized by the close proximity of Asp102.

The binding of OMTKY3 to PPE perturbs the proton affinity of an ionizable group on PPE, presumably His57, and the resulting proton linkage has also been studied. This proton linkage is the result of the enzyme mechanism, which requires that the histidine side chain in the active site be unprotonated in the complex between the enzyme and substrate. The relevance of the thermodynamics to the enzyme mechanism will be discussed below. The theoretical approach required to analyze calorimetric data for a system with proton linkage has been published [7] and is reviewed here.

\section{THEORY}

\section{Proton linkage}

In order to analyze calorimetric data for a system in which the binding is linked to a protonation equilibrium, the contributions of all processes to the observed enthalpy must be delineated [7-9]. For a system in which a ligand, L, binds to an enzyme, E, which contains an ionizable group, there are four states of the enzyme which are in equilibrium: the free and unprotonated enzyme, E; the free, protonated enzyme, $\mathrm{E}^{+}$; the bound and unprotonated enzyme, EL; and the bound and protonated enzyme, $\mathrm{EL}^{+}$. The observed affinity constant is then given by:

$K_{\mathrm{obs}}=\frac{[\mathrm{EL}]+\left[\mathrm{EL}^{+}\right]}{\left([\mathrm{E}]+\left[\mathrm{E}^{+}\right]\right)[\mathrm{L}]}=K_{\mathrm{int}} \frac{1+K_{\mathrm{p}}^{\mathrm{c}} a_{\mathrm{H}^{+}}}{1+K_{\mathrm{p}}^{\mathrm{f}} a_{\mathrm{H}^{+}}}$

where $K_{\text {int }}$ is the intrinsic affinity constant (i.e. for the binding of the inhibitor to the neutral enzyme), $K_{\mathrm{p}}^{\mathrm{f}}$ is the proton affinity constant (i.e. $10^{\mathrm{pKa}}$ ) to the free enzyme, $K_{\mathrm{p}}^{\mathrm{c}}$ is the proton affinity constant to the enzyme in complex with the inhibitor, and $a_{\mathrm{H}^{+}}$is the proton activity (i.e. $10^{-\mathrm{pH}}$ ). Thus at low proton activity (high $\mathrm{pH}$ ) the observed binding constant will approach the intrinsic constant.

Because there is a change in the proton affinity constant, protons are either absorbed or released by the enzyme upon binding. In a constant $\mathrm{pH}$ buffer, these protons are either released or absorbed by buffer molecules with a concomitant heat effect from the ionization of the buffer. The observed binding enthalpy in a calorimetric experiment, $\Delta H_{\mathrm{obs}}$, will be:

$\Delta H_{\text {obs }}=\Delta H_{0}+\mathrm{N}_{\mathrm{H}^{+}} \Delta H_{\text {ion }}$

where $\mathrm{N}_{\mathrm{H}^{+}}$is the number of protons released by the buffer, $\Delta H_{\text {ion }}$ is the ionization enthalpy of the buffer, and $\Delta H_{0}$ is the binding enthalpy that would be observed in a buffer with a $\Delta H_{\text {ion }}$ of zero. 
The number of protons released by the buffer, $\mathrm{N}_{\mathrm{H}+}$, is equivalent to the number of protons bound by the enzyme and is given as the difference between the average number of protons bound to the enzyme in the complex, $\overline{\mathrm{H}}^{\mathrm{c}}$, and bound to the free enzyme, $\overline{\mathrm{H}}^{\mathrm{f}}$. The average number of protons bound to the enzyme depends, in turn, on the proton affinity constants in the free and complexed proteins, $K_{\mathrm{p}}^{\mathrm{f}}$ and $K_{\mathrm{p}}^{\mathrm{c}}$, and the proton activity.

$\mathrm{N}_{\mathrm{H}^{+}}=\overline{\mathrm{H}}^{\mathrm{c}}-\overline{\mathrm{H}}^{\mathrm{f}}=\frac{K_{\mathrm{p}}^{\mathrm{c}} a_{\mathrm{H}^{+}}}{1+K_{\mathrm{p}}^{\mathrm{c}} a_{\mathrm{H}^{+}}}-\frac{K_{\mathrm{p}}^{\mathrm{f}} a_{\mathrm{H}^{+}}}{1+K_{\mathrm{p}}^{\mathrm{f}} a_{\mathrm{H}^{+}}}$

The value of $\Delta H_{0}$ reflects both the intrinsic binding enthalpy, i.e. the enthalpy of binding the inhibitor to the un-ionized enzyme, $\Delta H_{\text {int }}$, and the enthalpy associated with changes in the ionization state of the protein. The enthalpy changes associated with changes in the ionization state of the protein is given the difference between the enthalpy of protonating the complex $\left(\overline{\mathrm{H}}^{\mathrm{c}} \bullet \Delta H_{\mathrm{p}}^{\mathrm{c}}\right)$ and the enthalpy of protonating the free enzyme $\left(\overline{\mathrm{H}}^{\mathrm{f}} \bullet \Delta H_{\mathrm{p}}^{\mathrm{f}}\right)$ where $\Delta H_{\mathrm{p}}^{\mathrm{c}}$ and $\Delta H_{\mathrm{p}}^{\mathrm{f}}$ are the enthalpy of protonation of the complexed and free enzyme. Thus $\Delta H_{0}$ is given as:

$$
\begin{aligned}
\Delta H_{o} & =\Delta H_{\text {int }}+\overline{\mathrm{H}}^{\mathrm{f}} \Delta H_{\mathrm{p}}^{\mathrm{f}}+\overline{\mathrm{H}}^{\mathrm{c}} \Delta H_{\mathrm{p}}^{\mathrm{c}} \\
& =\Delta H_{\text {int }}-\overline{\mathrm{H}}^{\mathrm{f}} \Delta H_{\mathrm{p}}^{\mathrm{f}}+\overline{\mathrm{H}}^{\mathrm{c}}\left(\Delta H_{\mathrm{p}}^{\mathrm{f}}+\delta \Delta H_{\mathrm{p}}\right) \\
& =\Delta H_{\text {int }}+\mathrm{N}_{\mathrm{H}^{+}} \Delta H_{\mathrm{p}}^{\mathrm{f}}+\overline{\mathrm{H}}^{\mathrm{c}} \delta \Delta H_{\mathrm{p}}
\end{aligned}
$$

where $\delta \Delta H_{\mathrm{p}}$ is the difference in the enthalpy of protonation of the ionizable group on the protein in the complex relative to the free enzyme. Combining equations 2 and 4 yields:

$\Delta H_{\text {obs }}=\Delta H_{\text {int }}+\mathrm{N}_{\mathrm{H}^{+}}\left(\Delta \mathrm{H}_{\mathrm{p}}^{\mathrm{f}}+\Delta \mathrm{H}_{\text {ion }}\right)+\overline{\mathrm{H}}^{\mathrm{c}} \delta \Delta \mathrm{H}_{[} \mathrm{p}$

which illustrates that, even if the ionization enthalpy of the buffer and the protonation enthalpy of the protein are equal and opposite, the observed $\Delta H$ will not equal the intrinsic $\Delta H$ unless there is no change in the protonation enthalpy upon binding.

The temperature derivative of equation 6 yields the observed heat capacity change, $\Delta C_{\mathrm{p}, \mathrm{obs}}$, which will also depend upon the $\mathrm{pH}$ and buffer. The full expression for this derivative has been given previously [7]. The important thing to note is that the observed $\Delta H$ will depend upon temperature, $\mathrm{pH}$, and buffer, and that experiments performed as a function of these variables can be analyzed globally to determine the intrinsic binding energetics $\left(\Delta G_{\text {int }}^{\circ}, \Delta H_{\text {int }}\right.$ and $\left.\Delta C_{\text {p,int }}\right)$ as well as the energetics associated with the protonation of the protein including the $\mathrm{p} K_{\mathrm{a}}$ values in both the free enzyme and the complex and the enthalpies of protonation in both these states.

\section{Structural energetics calculations}

The procedures for estimating the thermodynamics of binding from structural data have been detailed previously $[6,10-12]$ and are only summarized here. The thermodynamics calculated from the structure are expected to correspond to the intrinsic energetics, but the 'int' subscript is omitted below for clarity.

The $\Delta C_{\mathrm{p}}$ of binding is expected to be proportional to the changes in apolar and polar accessible surface areas, $\Delta A_{\text {ap }}$ and $\Delta A_{\mathrm{p}}$, where the coefficients have been determined empirically from model compound dissolution data [13,14], Accessible surface areas are given in units of $\AA^{2}$.

$\Delta C_{\mathrm{p}}=1.88 \Delta A_{\mathrm{ap}}-1.09 \Delta A_{\mathrm{p}}$

The intrinsic $\Delta H^{\circ}$ of binding is likewise proportional to accessible surface area changes. The $\Delta H^{\circ}$ is estimated at $60^{\circ} \mathrm{C}(333 \mathrm{~K})$ because the parameters are derived from protein unfolding data $[15,16]$.

$\Delta H\left(60^{\circ} \mathrm{C}\right)=-35.3 \Delta A_{\text {ap }}+131 \Delta A_{\text {pol }}$

The intrinsic $\Delta S^{\circ}$ of binding primarily has contributions from three terms: restructuring of water in the solvation shell [17,18], loss of conformational entropy of side chains (and backbone) [19,20], and a 'mixing' entropy related to the loss in translational and rotational degrees of freedom upon binding 
[21,22], The overall, intrinsic $\Delta S^{\circ}$ at $25^{\circ} \mathrm{C}(298 \mathrm{~K})$ is:

$\Delta S=\Delta C_{\mathrm{p}} \ln \left(\frac{298}{385}\right)+\sum_{i} S_{\mathrm{sc}}^{\mathrm{o}} \frac{\Delta A_{\mathrm{tot}, \mathrm{i}}}{A_{\mathrm{max}, \mathrm{i}}}+R \ln \left(\frac{1}{55}\right)$

The first term gives the contribution of solvent reorganization which is assumed to be directly proportional to $\Delta C_{\mathrm{p}}[17,18]$, The second term gives the contribution of changes in conformational entropy of the side chains due to loss of accessible rotomeric states. The maximum conformational entropy of the $i$ th side chain, $S_{\mathrm{sc}}^{\circ}$, has been estimated computationally for each amino acid type [19]. The maximum conformational entropy will occur when the residue is full exposed to solvent, having an accessible surface area of $A_{\max }$; the side chain has no conformational entropy (i.e. only one rotomeric state) if it is fully buried. It is assumed that the conformational entropy varies linearly with the degree of exposure of the side chain $[6,11]$, The final term is for the mixing entropy and is simply the cratic entropy for a bimolecular association in aqueous solution $[23,24]$.

The intrinsic $\Delta G^{\circ}$ of binding can be calculated as a function of temperature using equations $6-8$ and the standard relationship:

$\Delta G^{\circ}=\Delta H_{\mathrm{R}}^{\circ}-T \Delta S_{\mathrm{R}}^{\circ}+\Delta C_{\mathrm{p}}\left[\left(T-T_{\mathrm{R}}\right)-T \ln \left({ }^{T} T_{T_{\mathrm{R}}}\right)\right]$

where $T_{\mathrm{R}}$ is any convenient reference temperature and $\Delta H_{\mathrm{R}}^{\circ}$ and $\Delta S_{\mathrm{R}}^{\circ}$ are the enthalpy and entropy changes at that temperature. The structural information required is the changes in apolar and polar accessible surface areas upon binding and, for calculating conformational entropy, the contribution of each amino acid side chain to these changes.

For the binding of OMTKY3 to PPE, $1130 \AA^{2}$ of apolar surface area and $660 \AA^{2}$ of polar surface area are calculated to be buried upon formation of the complex [6]. These values, in conjunction with the identities of the amino acid side chains involved were used with equations 6-9 to estimate the intrinsic energetics of binding. These estimates are summarized in Table 1.

Table 1 Structural energetics prediction of the intrinsic thermodynamics of binding of OMTKY3 to PPE at 298 K [6]

\begin{tabular}{lc} 
Parameter & Predicted value \\
\hline$\Delta C_{\mathrm{p}}$ & $-1.4 \mathrm{~J} / \mathrm{K} / \mathrm{mol}$ \\
$\Delta H^{\circ}$ & $2.3 \mathrm{~kJ} / \mathrm{mol}$ \\
$\Delta S^{\circ}$ & $190 \mathrm{~J} / \mathrm{K} / \mathrm{mol}$ \\
$\Delta G^{\circ}$ & $-54 \mathrm{~kJ} / \mathrm{mol}$ \\
\hline
\end{tabular}

\section{RESULTS AND DISCUSSION}

Isothermal titration calorimetry experiments were performed over a range of temperature and $\mathrm{pH}$ in buffers with various ionization enthalpies. One hundred and forty-three datum points, from 19 different experimental conditions, were fit globally using equations $2-5$ to determine the intrinsic binding energetics and the energetics of protonation as given in Tables 2 and 3. Because of the high affinity of OMTKY 3 for PPE, it is not possible to determine $K$ experimentally in the calorimeter. The value of $K$ has been measured from residual enzyme activity [25], and the literature value was used to determine the intrinsic $\Delta G^{\circ}$ and, with the fitted $\Delta H^{\circ}$ value, $\Delta S^{\circ}[6]$.

Table 2 Experimentally derived intrinsic thermodynamics of binding of OMTKY3 to PPE at 298 K [6]

\begin{tabular}{lc} 
Parameter & Fitted value \\
\hline$\Delta C_{\mathrm{p}}$ & $-1.1 \pm 0.1 \mathrm{~J} / \mathrm{K} / \mathrm{mol}$ \\
$\Delta H^{\circ}$ & $-2.5 \pm 1.0 \mathrm{~kJ} / \mathrm{mol}$ \\
$\Delta S^{\circ}$ & $195 \pm 4 \mathrm{~J} / \mathrm{K} / \mathrm{mol}$ \\
$\Delta G^{\circ}$ & $-60.6 \pm 0.5 \mathrm{~kJ} / \mathrm{mol}$
\end{tabular}


Table 3 Experimentally determined thermodynamics of protonation for the binding of OMTKY3 to PPE [6]. The ionizable group is assumed to be His 57 of PPE

\begin{tabular}{lc} 
Parameter & Fitted value \\
\hline $\mathrm{p} K_{\mathrm{a}}^{\mathrm{f}}$ & $6.7 \pm 0.1$ \\
$\mathrm{p} K_{\mathrm{a}}^{\mathrm{c}}$ & $5.2 \pm 0.1$ \\
$\Delta H_{\mathrm{p}}^{\mathrm{f}}$ & $-27.9 \pm 2.6$ \\
$\delta \Delta H_{\mathrm{p}}$ & $12.5 \pm 3.5$ \\
$\Delta S_{\mathrm{p}}^{\mathrm{f}}$ & $34.7 \pm 8.9$ \\
$\delta \Delta S_{\mathrm{p}}$ & $13.3 \pm 17.5$ \\
\hline
\end{tabular}

\section{Binding energetics}

Comparison of the experimental and predicted energetics in Tables 1 and 2 shows that the Structural Energetics calculations perform very well for the OMTKY3/PPE system. The agreement between the predicted and experimental values of $\Delta C_{\mathrm{p}}$ and $\Delta S^{\circ}$ is very good. The predicted and experimental values of $\Delta H^{\circ}$ are, in fact, of opposite sign, but both are very small values and the $\Delta S^{\circ}$ dominates the $\Delta G^{\circ}$ of binding at $25^{\circ} \mathrm{C}$ as predicted. The negligible $\Delta H^{\circ}$ arises from compensation of the favorable $\Delta H^{\circ}$ of intramolecular hydrogen bond formation and the unfavorable $\Delta H^{\circ}$ of burying the hydrophobic groups in the interface. Nevertheless, the error in predicting $\Delta G^{\circ}$ arises primarily from the error in predicting $\Delta H^{\circ}$.

\section{Proton binding energetics}

The proton binding energetics derived from the calorimetric data are significant in understanding the mechanism of serine protease function. The $\mathrm{p} K_{\mathrm{a}}$ of the histidine in the free enzyme is 6.7. Consequently, at $\mathrm{pH} 7$, about $33 \%$ of the enzyme molecules will have a protonated histidine. Because a protonated histidine cannot accept the proton from the serine hydroxyl, these molecules are catalytically inactive. In order for proteolysis to be efficient, the enzyme must be 'activated' upon binding of substrate (or inhibitor) which requires a decrease in the proton affinity of the histidine so that it is in the unprotonated state initially. This presents an additional problem, however, because the enzyme still needs to transfer a proton from the serine hydroxyl to the histidine which requires an increase in the proton affinity of the histidine (or a decrease in the proton affinity of the serine). The protonation energetics provide some clues as to how the enzyme manages to fulfil these two, apparently mutually exclusive tasks.

Both $\mathrm{p} K_{\mathrm{a}}^{\mathrm{f}}$ and $\Delta H_{\mathrm{p}}^{\mathrm{f}}$ are typical for the protonation of exposed histidine residues [26]. Upon formation of the complex, the $\mathrm{p} K_{\mathrm{a}}$ drops to 5.2, decreasing the proton affinity as required so that the histidine is free to accept a proton from the serine hydroxyl. The decrease in proton affinity arises from a decrease in magnitude of the favorable $\Delta H_{\mathrm{p}}$.

Examination of the structures of the free enzyme and the enzyme in complex with inhibitor show that the histidine side chain largely is exposed in the free enzyme but is fully buried in the complex. The exposure of the histidine residue in the free enzyme allows for the positive charge on the protonated form to be well solvated by water. However, solvent water cannot interact with the protonated form of histidine in the complex so that solvent must be stripped from the proton when the proton is transferred from solvent to the histidine upon protonation of the complex. The enthalpy of stripping this water is likely the source of the enthalpic penalty observed in protonating the enzyme in complex relative to the free enzyme.

In contrast to protons originating from solvent, the proton on the serine hydroxyl is fully buried in the complex and thus already desolvated. Consequently, there is no desolvation penalty for the transfer of this proton to the histidine side chain. The fact that the active site of the enzyme is buried in the complex thus helps to make the protonation of the histidine more specific for the proton from the serine by adding a penalty for protonation from solvent. The need to raise the proton affinity of the histidine in order to affect transfer from the serine hydroxyl requires additional contributions, possibly from a low barrier hydrogen bond to Asp 102, but the energetics of protonation add considerably to our understanding of proton management in serine proteases. 


\section{Phosphate binding}

We noted previously [6] that the binding enthalpy was dependent on buffer concentration for some buffers, namely phosphate. These buffers were excluded in the original analysis of the proton linkage and intrinsic binding energetics, but the results suggest that phosphate may interact specifically with the PPE/ OMTKY3 complex. Indeed, the crystal structure of the complex between OMTKY3 and another serine protease, Streptomyces grisseus Protease B, shows a phosphate bound to the complex which is ligated by residues from both proteins [27].

In order to investigate further the interaction of phosphate with the protease-inhibitor complex, we have studied the binding over a range of phosphate concentrations at $25^{\circ} \mathrm{C}$ and at $\mathrm{pH} 6.0$. If phosphate binds to the complex with affinity $K_{\text {phos }}$ and a binding enthalpy of $\Delta H_{\text {phos }}$, then $\Delta H_{\text {obs }}$ for the binding of OMTKY3 to PPE is given as:

$\Delta H_{\text {obs }}=\Delta H_{0}+\frac{K_{\text {phos }}\left[\mathrm{P}_{\mathrm{i}}\right]}{1+K_{\text {phos }}\left[\mathrm{P}_{\mathrm{i}}\right]} \Delta H_{\text {phos }}$

where $\Delta H_{0}$ is the $\Delta H$ of binding that would be observed at $\mathrm{pH} 6$ in a buffer with a $\Delta H_{\text {ion }}$ identical to phosphate but which does not interact with the complex, and $\left[\mathrm{P}_{\mathrm{i}}\right]$ is the concentration of inorganic phosphate.

Data collected between 25 and $250 \mathrm{~mm}$ phosphate concentration were fitted to equation 10 as shown in Fig. 1. The fit of these data suggest that phosphate binds to the OMTKY3/PPE complex with a weak binding constant of about $10 \pm 3$ with a $\Delta H^{\circ}$ of binding of $20 \pm 2 \mathrm{~kJ} / \mathrm{mol}$. Thus phosphate binding is entropically driven. The weak binding constant is consistent with the observation that the binding affinity of OMTKY3 to PPE does not depend on phosphate concentration (Michael J. Laskowski Jr., personal communication). Additional data as a function of temperature and $\mathrm{pH}$ will be required to better understand the molecular basis for phosphate recognition of proteins.

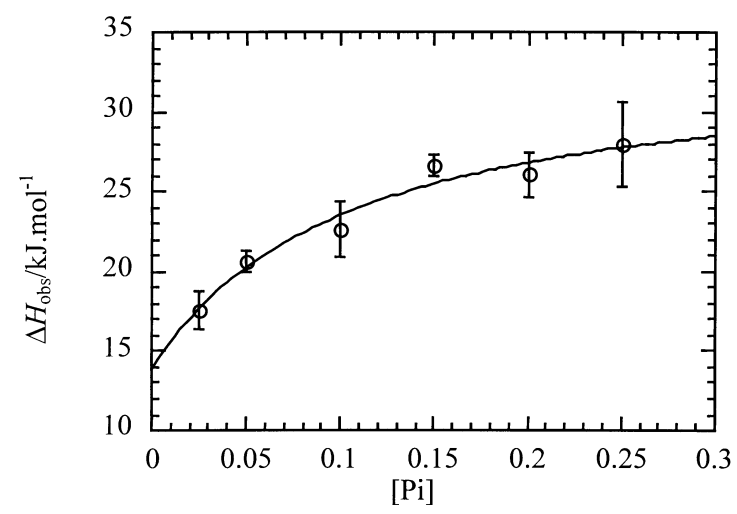

Fig. 1 Observed enthalpy of binding OMTKY3 to PPE as a function of phosphate concentration at 298 K, pH 6.0.

\section{CONCLUSIONS}

Molecular recognition between proteins reflects both the intrinsic interactions between atomic groups of the involved proteins and the effects of environmental factors such as temperature and $\mathrm{pH}$. We have characterized many of these factors for the binding of the inhibitor OMTKY 3 to the serine protease PPE.

The intrinsic interaction, that is the interaction between the proteins when the ionizable active site histidine of the PPE is unprotonated, is characterized by a negligible $\Delta H^{\circ}$ and a large favorable $\Delta S^{\circ}$. These energetics are consistent with the structural model which shows significant burial of hydrophobic surface. However, the hydrophobic effect is not the sole contributor to binding and the role of hydrogen bonds, particularly to the $\Delta H^{\circ}$ of binding, is significant [6]. The binding thermodynamics are well predicted by structural energetic calculations as has been described [6]. The excellent agreement between the predicted and experimental binding thermodynamics suggests the structural energetic calculations 
might have considerable utility for structure based design of proteins and protein inhibitors as has been shown recently

The effects of environmental factors, namely $\mathrm{pH}$ and the concentration of small inorganic molecules, provide a means of regulating protein-protein interactions within the cell. These effects are mediated through linked binding [28]. The effects of proton linkage in the binding of OMTKY3 to PPE reveal the importance of having an active site which is inaccessible to water when substrate is bound. This water inaccessibility makes the histidine more selective for transfer of a proton from the active site histidine rather than from buffer.

While phosphate does not appear to be important for the biological regulation of serine protease inhibition, the OMTKY3-PPE system also can serve as a model for the binding of phosphate to proteins. Preliminary data on this interaction suggests that it is entropically driven at $25^{\circ} \mathrm{C}$.

\section{ACKNOWLEDGEMENTS}

This work was supported by the National Science Foundation.

\section{REFERENCES}

1 N. Horton, M. Lewis. Protein Sci. 1, 169 (1992).

2 G. Verkhivker, K. Appelt, S. T. Freer, J. E. Villafranca. Protein Eng 8, 677 (1995).

3 S. Vajda, M. Sippl, J. Novotny. Curr. Opin. Struct Biol. 7, 222 (1997).

4 Z. Weng, C. Delisi, S. Vajda. Protein Sci. 6, 1976 (1997).

5 N. Froloff, A. Windemuth, B. Honig. Protein Sci. 6, 1293 (1997).

6 B. M. Baker, K. P. Murphy. J. Mol. Biol. 268, 557 (1997).

7 B. M. Baker, K. P. Murphy. Biophys. J. 71, 2049 (1996).

8 M. R. Eftink, A. C. Anusiem, R. L. Biltonen. Biochemistry 22, 3884 (1983).

9 M. Eftink, R. Biltonen. Thermodynamics of Interacting Biological Systems, p. 343. Academic Press, San Diego (1980).

10 K. P. Murphy, D. Xie, K. C. Garcia, L. M. Amzel, E. Freire. Proteins 15, 113 (1993).

11 J. Gómez, E. Freire. J. Mol. Biol. 252, 337 (1995).

12 B. M. Baker, K. P. Murphy. Meth. Enzymol. 295, 294 (1998).

13 K. P. Murphy, S. J. Gill. J. Mol. Biol. 222, 699 (1991).

14 K. P. Murphy, E. Freire. Adv. Protein Chem. 43, 313 (1992).

15 D. Xie, E. Freire. Proteins 19, 291 (1994).

16 K. P. Murphy, E. Freire. Pure Appl. Chem. 65, 1939 (1993).

17 R. L. Baldwin. Proc. Natl. Acad. Sci. USA 83, 8069 (1986).

18 K. P. Murphy, P. L. Privalov, S. J. Gill. Science 247, 559 (1990).

19 K. H. Lee, D. Xie, E. Freire, L. M. Amzel. Proteins 20, 68 (1994).

20 T. P. Creamer, G. D. Rose. Proc. Natl. Acad. Sci. USA 89, 5937 (1992).

21 K. P. Murphy, D. Xie, K. S. Thompson, L. M. Amzel, E. Freire. Proteins 18, 63 (1994).

22 L. M. Amzel. Proteins 28, 144 (1997).

23 R. W. Gurney. Ionic Processes in Solution. McGraw-Hill Book Company Inc., New York (1953).

24 W. Kauzmann. Adv. Protein Chem. 14, 1 (1959).

25 M. W. Empie, M. Laskowski Jr. Biochemistry 21, 2274 (1982).

26 J. J. Christensen, L. D. Hansen, R. M. Izatt. Handbook of Proton Ionization Heats and Related Thermodynamic Quantities. John Wiley \& Sons, New York (1976).

27 K. Huang, W. Lu, S. Anderson, M. Laskowski Jr, M.N.G. James. Protein Sci. 4, 1985 (1995).

28 J. Wyman, S. J. Gill. Binding and Linkage: the Functional Chemistry of Biological Macromolecules. University Science Books, Mill Valley (1990). 